\section{The role of contrast-enhanced ultrasonography in image-guided liver ablations}

\author{
Lorenzo Carlo Pescatori ${ }^{1}$, Luca Maria Sconfienza ${ }^{2,3}$, Giovanni Mauri ${ }^{2,4}$ \\ ${ }^{1}$ Postgraduate School in Radiodiagnostics, Università degli Studi di Milano, Milan; ${ }^{2}$ Radiology \\ Unit, Policlinico San Donato Research Hospital, Milan; ${ }^{3}$ Department of Biomedical Sciences \\ for Health, Università degli Studi di Milano, Milan; ${ }^{4}$ European Institute of Oncology, Milan, \\ Italy
}

We read with great interest the paper by Kim et al. [1] entitled "Local ablation therapy with contrast enhanced ultrasonography for hepatocellular carcinoma: a practical review," recently published in Ultrasonography. We think that contrast-enhanced ultrasonography (CEUS), together with the development of reliable navigation systems, is likely to represent one of the most important advances in image-guided ablations in recent years. Thus, we offer some considerations on the topic.

Percutaneous ablations, performed with different technologies, are increasingly used to treat a variety of conditions, including liver, kidney, and even neck tumors [2,3]. Whatever the technology, most procedures are performed under sonographic guidance, which provide a reliable, real-time, and cheap guidance method even in extremely complex conditions. However, in some cases, lesions may be not clearly detectable on ultrasonography, due to low conspicuity or when visibility is impaired by bones or aerated organs (i.e., the lungs and bowel). In these cases, the use of more complex guidance modalities, such as computed tomography (CT), magnetic resonance imaging (MRI), or even positronemitting tomography (PET), have been reported [3]. However, all these methods imply an increased level of technical difficulty, longer procedure duration, and higher costs. Kim et al. [1] highlighted how the use of CEUS may help in performing liver ablations in cases of lesions not clearly visible on standard ultrasonography. We consider the intra-procedural use of CEUS of paramount importance for image-guided ablations. In our experience, CEUS has allowed for the immediate retreatment of patients with incomplete ablations. This implied a dramatic decrease in rate of repeat visit and repeat ablation, thus also remarkably reducing the overall costs per patient [4]. Unlike Kim et al. [1], we generally perform post-ablation CEUS after 5-7 minutes, as soon as the gas that developed during the procedure has disappeared. At 20-40 minutes, a peripheral hyperemic halo may be present, potentially mimicking the presence of a residual peripheral tumor.

However, even CEUS may not be sufficient to fully depict the lesion with full conspicuity in certain patients. Under these conditions, fusion imaging between ultrasonography and pre-acquired $\mathrm{CT}_{\text {, }}$ $\mathrm{MRI}$, or PET images, together with virtual navigation, may represent a valuable option [5]. In our experience, we were able to achieve correct targeting of lesions not thoroughly detectable at ultrasonography or CEUS in more than $90 \%$ of cases, thanks to the availability of a fusion system [5].

In conclusion, we think that CEUS, virtual navigation, and fusion systems may enable the application of minimally-invasive image-guided procedures to a larger population, potentially avoiding major surgery in a selected subset of patients.

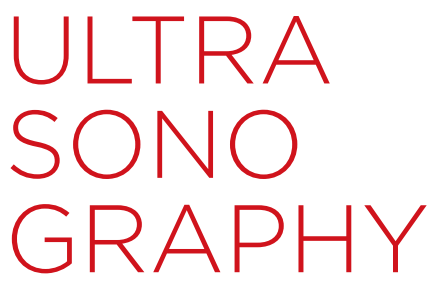

\section{LETTER}

http://dx.doi.org/10.14366/usg. 15046 pISSN: 2288-5919 - elSSN: 2288-5943 Ultrasonography 2016;35:87-88

Received: August 13, 2015 Accepted: August 18, 2015

Correspondence to: Lorenzo Carlo Pescatori, MD, Department of Radiology, IRCCS Policlinico San Donato, Piazza Edmondo Malan 1, 20097, San Donato Milanese, Milan, Italy

Tel. +39-0252774641

Fax. +39-0252774626

E-mail: lorenzo.carlo.pescatori@gmail. com

\footnotetext{
This is an Open Access article distributed under the terms of the Creative Commons Attribution NonCommercial License (http://creativecommons.org/ licenses/by-nc/3.0/) which permits unrestricted noncommercial use, distribution, and reproduction in any medium, provided the original work is properly cited.
}

Copyright (C) 2016 Korean Society of Ultrasound in Medicine (KSUM)

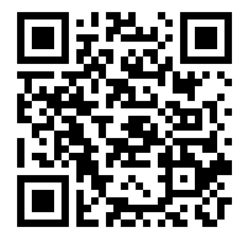

How to cite this article:

Pescatori LC, Sconfienza LM, Mauri G. The role of contrast-enhanced ultrasound in imageguided liver ablations. Ultrasonography. 2016 Jan;35(1):87-88. 


\section{Conflict of Interest}

No potential conflict of interest relevant to this article was reported.

ORCID: Lorenzo Carlo Pescatori: http://orcid.org/0000-0001-8055-4801; Luca Maria Sconfienza: http://orcid.org/0000-0003-0759-8431; Giovanni Mauri: http://orcid.org/0000-0003-4726-2692

\section{References}

1. Kim TK, Khalili K, Jang HJ. Local ablation therapy with contrastenhanced ultrasonography for hepatocellular carcinoma: a practical review. Ultrasonography 2015 Jun 24 [Epub]. http://dx.doi.org/ 10.14366/usg.15018.

2. Shyn PB, Mauri G, Alencar RO, Tatli S, Shah SH, Morrison PR, et al. Percutaneous imaging-guided cryoablation of liver tumors: predicting local progression on 24-hour MRI. AJR Am J Roentgenol 2014;203:W181-W191.

3. Shyn PB, Tatli S, Sahni VA, Sadow CA, Forgione K, Mauri G, et al. $\mathrm{PET} / \mathrm{CT}$-guided percutaneous liver mass biopsies and ablations: targeting accuracy of a single $20 \mathrm{~s}$ breath-hold PET acquisition. Clin Radiol 2014;69:410-415.

4. Mauri G, Porazzi E, Cova L, Restelli U, Tondolo T, Bonfanti M, et al. Intraprocedural contrast-enhanced ultrasound (CEUS) in liver percutaneous radiofrequency ablation: clinical impact and health technology assessment. Insights Imaging 2014;5:209-216.

5. Mauri G, Cova L, De Beni S, lerace T, Tondolo T, Cerri A, et al. Realtime US-CT/MRI image fusion for guidance of thermal ablation of liver tumors undetectable with US: results in 295 cases. Cardiovasc Intervent Radiol 2015;38:143-151. 